\title{
MUTUAL INDUCTANCE OF ANY TWO CIRCLES
}

\author{
By Chester Snow
}

\section{ABSTRACT}

A formal expansion is derived for the magnetic flux through any circle. A similar expansion is obtained for the magnetic potential due to a unit current in another circle. Combining the two gives a formal expansion for the mutual inductance of the two circles. Application is made to two cases (1) where the circles are parallel, (2) where their axes intersect.

\section{CONTENTS}

I. Introduction

II. Symbolic expression for magnetic flux through a circle

III. Symbolic expression for the magnetic potential of a circular current

IV. Combination of II and III for the mutual inductance of the two circles.

1. Case of parallel circles

2. Special case where the axes of the two circles intersect.

(a) The axes intersect at center of one circle........ 540

(b) Circles coaxial_._._._._. 541

(c) Circles concentric, but not necessarily coaxial__._. 541

V. Convergence of the expansion . . .

\section{INTRODUCTION}

In certain absolute measurements it is necessary to compute with precision the electromagnetic forces exerted between two currentcarrying solenoids, or the current induced in one by changes of that in the other. Both problems require a formula for the mutual inductance of the two, which is generally to be derived by integrating the expression for the mutual inductance between two circles. The fundamental importance of the two circular elements is evident. When the circles are not only parallel but coaxial, their mutual inductance is given exactly by Maxwell's formula.

In 1916 Butterworth ${ }^{1}$ derived a formula covering (partially) the case of two parallel circles. In the present paper is presented a general formal expression for any two circles which leads readily to Butterworth's formula as a special case, and also to a fairly simple expansion in zonal harmonics for the mutual inductance of two circles whose 
axes intersect at a finite point (equation (23) below). From it may be derived simple formulas for the mutual inductance and torque between two solenoids whose axes intersect, one lying entirely within the other, as in certain types of current balance.

The starting point of these developments is a formal expression here obtained for the magnetic flux through a circle of radius $a_{2}$, the field being the negative gradient of a scalar potential $\Omega\left(x_{2}, y_{2}, z_{2}\right)$ at the point $P_{2}\left(x_{2}, y_{2}, z_{2}\right)$, which is the center of the circle. It is

$$
M=-\mathcal{S} \mathcal{S} D_{\mathrm{n}_{2}} \Omega d S=-2 \pi a_{2} J_{1}\left(a_{2} D_{\mathrm{n}_{2}}\right) \Omega\left(x_{2}, y_{2}, z_{2}\right)
$$

where $J_{1}$ is Bessel's function of the first order, the symbol $D_{\mathrm{n}_{2}}$ designating the space derivative in the direction normal to the plane of the circle - at the center.

Thus

where

$$
J_{1}\left(a_{2} D_{\mathrm{n}_{2}}\right) \Omega=\sum_{\mathrm{k}=0}^{\infty} \frac{(-1)^{\mathrm{k}}\left(\frac{a_{2}}{2}\right)^{2 \mathrm{k}+1}}{k !(k+1) !} D_{\mathrm{n}_{2}}{ }^{2 \mathrm{k}+1} \Omega
$$

$$
D_{\mathrm{n}_{2}} \Omega \equiv\left(l_{2} D_{\mathbf{x}_{2}}+m_{2} D_{\mathbf{y}_{2}}+n_{2} D_{\mathrm{z}_{2}}\right) \Omega
$$

the direction cosines of the normal to the circle being $l_{2}, m_{2}, n_{2}$.

If the field $\Omega\left(x_{2}, y_{2}, z_{2}\right)$ is that produced by a unit current in a circle of radius $a_{1}$ center at $P_{1}\left(x_{1}, y_{1}, z_{1}\right)$, it is then shown that

$$
\Omega\left(x_{2}, y_{2}, z_{2}\right)=2 \pi a_{1} J_{1}\left(a_{1} D_{\mathrm{n}_{1}}\right) \frac{1}{r}
$$

where $r$ is the distance between centers. The mutual inductance between the two circles is then given (formally) by

$$
M=-4 \pi^{2} a_{1} a_{2} J_{1}\left(a_{2} D_{\mathrm{n}_{2}}\right) J_{1}\left(a_{1} D_{\mathrm{n}_{1}}\right) \frac{1}{r}
$$

By obtaining expansions for $\frac{1}{r}$ which are suitable from the point of view of performing the infinite series of differentiations here indicated, a variety of series formulas may be obtained.

\section{SYMBOLIC EXPRESSION FOR THE MAGNETIC FLUX THROUGH A CIRCLE}

Let $\Omega(x, y, z)$ be a scalar point function which may be expanded by Taylor's (three-dimentional) theorem about the point $x_{0}, y_{0}, z_{0}$. This expansion may be written symbolically if we let

$$
\begin{gathered}
x=x_{0}+x^{\prime}, y=y_{\mathrm{o}}+y^{\prime}, z=z_{\mathrm{o}}+z^{\prime} \\
\Omega(x, y, z)=\Omega\left(x_{\mathrm{o}}+x^{\prime}, y_{\mathrm{o}}+y^{\prime}, z_{\mathrm{o}}+z^{\prime}\right)=e^{\mathrm{x}^{\prime} \mathrm{D}_{\mathrm{x}_{\mathrm{o}}}+\mathrm{y}^{\prime} \mathbf{D}_{\mathbf{y}_{\mathrm{o}}}+z^{\prime} \mathrm{D}_{\mathrm{zo}}} \Omega\left(x_{\mathrm{o}}, y_{\mathrm{o}}, z_{\mathrm{o}}\right)
\end{gathered}
$$


Consider a circle of radius $a$ whose center is at $x_{0}, y_{0}, z_{0}$ and whose plane is perpendicular to the $z$ axis. Let $u$ denote the surface integral of $\Omega$ over this circle. Then

$$
\begin{aligned}
u & =\int_{-\mathrm{a}}^{\mathrm{a}} d x^{\prime} \int_{-\sqrt{\mathrm{a}^{2}-\mathrm{x}^{\prime 2}}}^{\sqrt{\mathrm{a}^{2}-\mathrm{x}^{\prime 2}}} d y^{\prime} \Omega\left(x_{0}+x^{\prime}, y_{\mathrm{o}}+y^{\prime}, z_{\mathrm{o}}+z^{\prime}\right) \\
& =\int_{-\mathrm{a}}^{\mathrm{a}} d x^{\prime} \int_{\sqrt{\mathrm{a}^{2}-\mathrm{x}^{\prime 2}}}^{\sqrt{\mathrm{a}^{2}-\mathrm{x}^{\prime 2}}} d y^{\prime} e^{\mathrm{x}^{\prime}} \mathrm{D}_{\mathrm{x}_{\mathrm{o}}}+\mathrm{y}^{\prime} \mathrm{D}_{\mathbf{y}_{\mathrm{o}}} \Omega\left(x_{0}, y_{\mathrm{o}}, z_{\mathrm{o}}\right)
\end{aligned}
$$

In this expression the symbolic operator $e^{\mathrm{x}^{\prime} \mathbf{D}_{\mathrm{x}_{\mathrm{o}}}+\mathrm{y}^{\prime} \mathrm{D}_{y_{\mathrm{o}}}} \Omega\left(x_{0}, y_{\mathrm{o}}, z_{\mathrm{o}}\right)$ is merely the abbreviated form of the series of operations indicated by

$$
e^{\mathrm{x}^{\prime} \mathrm{D}_{\mathrm{x}_{\mathrm{o}}}+\mathrm{y}^{\prime} \mathrm{D}_{\mathrm{y}_{\mathrm{o}}}} \Omega\left(x_{\mathrm{o}}, y_{\mathrm{o}}, z_{\mathrm{o}}\right)=\sum_{\mathrm{n}=\mathrm{o}}^{\infty} \frac{\left(x^{\prime} D_{\mathrm{x}_{\mathrm{o}}}+y^{\prime} D_{\mathrm{y}_{\mathrm{o}}}\right)^{\mathrm{n}}}{n !} \Omega\left(x_{\mathrm{o}}, y_{\mathrm{o}}, z_{\mathrm{o}}\right)
$$

where each term $\left(x^{\prime} D_{\mathbf{x}_{0}}+y^{\prime} D_{\mathbf{y}_{0}}\right)^{\mathrm{n}} \Omega\left(x_{0}, y_{0}, z_{0}\right)$ is to be expanded formally by the binominal theorem. The result of using this formal expansion equation (3) in the integral, equation (2), may be found by noting that the symbols $D_{\mathbf{x}_{0}}$ and $D_{\mathbf{y}_{0}}$ behave like constants as far as integration with respect to $x^{\prime}$ and $y^{\prime}$ is concerned. It may be written out from a consideration of the formal analogy between it and the integral $u_{\mathrm{o}}$ where

$$
u_{\mathrm{o}}=\int_{-\mathrm{a}}^{\mathrm{a}} d x^{\prime} \int_{-\sqrt{\mathrm{a}^{2}-\mathrm{x}^{\prime 2}}}^{\sqrt{\mathrm{a}^{2}-\mathrm{x}^{\prime 2}}} d y^{\prime} e^{\mathrm{x}^{\prime} \mathrm{A}+\mathrm{y}^{\prime} \mathrm{B}} \Omega_{\mathrm{o}}
$$

where $A, B$, and $\Omega_{\mathrm{o}}$ do not involve $x^{\prime}$ or $y^{\prime}$ and are, therefore, constants as far as this integration is concerned. This integral may be readily evaluated by choosing a new pair of rectangular axes $x^{\prime \prime}, y^{\prime \prime}$ in the same plane as before and with the same center such that

$$
x^{\prime \prime}=x^{\prime} \frac{A}{\sqrt{A^{2}+B^{2}}}+y^{\prime} \frac{B}{\sqrt{A^{2}+B^{2}}}
$$

In terms of these axes the expression for $u_{0}$ is

$$
\begin{aligned}
u_{0} & =\Omega_{0} \int_{-\mathrm{a}}^{\mathrm{a}} d x^{\prime \prime} \int_{-\sqrt{\mathrm{a}^{2}-\mathrm{x}^{\prime 2}}}^{\sqrt{\mathrm{a}^{2}-\mathrm{x}^{2}}} d y^{\prime \prime} e \mathrm{x}^{\prime \prime} \sqrt{\mathrm{A}^{2}+\mathrm{B}^{2}}=2 a^{2} \Omega_{\mathrm{o}} \int_{0}^{\pi} \sin ^{2} \theta e \sqrt{\mathrm{A}^{2}+\mathrm{B}^{2}} \cos \theta d \theta \\
& =2 a^{2} \Omega_{0} \sum_{\mathrm{n}=0}^{\infty} \frac{\left(a \sqrt{A^{2}+B^{2}}\right)^{\mathrm{n}}}{n !} \int_{0}^{\pi} \cos ^{\mathrm{n}} \theta \sin ^{2} \theta d \theta
\end{aligned}
$$


Now $\int_{0}^{\pi} \cos ^{n} \theta \sin ^{2} \theta d \theta=0$ if $n$ is odd

Hence

$$
=\frac{\pi}{2^{2 s+1}} \frac{(2 s) !}{s !(s+1) !} \text { if } n \text { is even; } n=2 s ; s=0,1,2,3 \cdots
$$

$$
\begin{aligned}
u_{\mathrm{o}} & =\pi a^{2} \sum_{\mathrm{s}=0}^{\infty} \frac{\left(\frac{a \sqrt{A^{2}+B^{2}}}{2}\right)^{2 s}}{s !(s+1) !} \Omega_{\mathrm{o}}=2 \pi a \frac{J_{1}\left(i a \sqrt{A^{2}+B^{2}}\right)}{i \sqrt{A^{2}+B^{2}}} \Omega_{\mathrm{o}} \\
& =\iint e^{\mathrm{x}^{\prime} \mathrm{A}+\mathrm{y}^{\prime} \mathrm{B}} \Omega_{\mathrm{o}} d S \text { integrated over the circular area. } J_{1}
\end{aligned}
$$

is Bessel's function of order one.

A comparison of this equation with the symbolic equation (2) ennables the latter to be put in the form

$$
\begin{aligned}
u \equiv \int \Omega(x, y, z) d S & =\pi a^{2} \sum_{\mathrm{s}=0}^{\infty} \frac{\left(\frac{a}{2}\right)^{2 \mathrm{~s}}\left(D_{\mathrm{x}_{\mathrm{o}}}^{2}+D_{\mathrm{y}_{\mathrm{o}}}^{2}\right)^{\mathrm{s}}}{s !(s+1) !} \Omega\left(x_{\mathrm{o}}, y_{\mathrm{o}}, z_{\mathrm{o}}\right) \\
& =2 \pi a \frac{J_{1}\left(i a \sqrt{D_{\mathrm{x}_{\mathrm{o}}}^{2}+D_{\mathrm{y}_{\mathrm{o}}}^{2}}\right)}{i \sqrt{D_{\mathrm{x}_{\mathrm{o}}}^{2}+D^{2}{ }_{\mathrm{y}_{\mathrm{o}}}}} \Omega\left(x_{\mathrm{o}}, y_{0}, z_{\mathrm{o}}\right)
\end{aligned}
$$

The meaning of $\sqrt{D_{x_{0}}^{2}+D_{y_{0}}^{2}}$ is merely an operator whose repetition is equivalent to the operator $D_{x_{0}}^{2}+D_{y_{0}}^{2}$, and since none but positive even "powers" of this operator occur here it need not be further defined.

The expression (6) assumes a particular interest in case the function $\Omega(x, y, z)$ satisfies Laplace's equation for in that case

$$
\left(D_{\mathrm{x}_{\mathrm{o}}}^{2}+D_{\mathrm{y}_{\mathrm{o}}}^{2}\right)^{\mathrm{s}} \Omega\left(x_{\mathrm{o}}, y_{\mathrm{o}}, z_{\mathrm{o}}\right)=(-1)^{\mathrm{s}} D_{\mathrm{z}_{\mathrm{o}}}^{2 \mathrm{~s}} \Omega\left(x_{0}, y_{\mathrm{o}}, z_{\mathrm{o}}\right)
$$

and (6) becomes

$$
\int \Omega d S=\pi a^{2} \sum_{s=0}^{\infty} \frac{(-1)^{\mathrm{s}}\left(\frac{a D_{z_{0}}}{2}\right)^{2 \mathrm{~s}}}{s !(s+1) !} \Omega\left(x_{0}, y_{0}, z_{0}\right)
$$

The surface integral of any harmonic point function $\Omega$, over any circle of radius $a$, is thus expressed in terms of the values which are assumed at its center by the function and its normal derivatives of even order. If $\Omega(x, y, z)$ is harmonic, then so is $D_{z_{0}} \Omega$. Hence, if we replace $\Omega$ by $D_{z_{0}} \Omega$ it becomes

$$
\begin{aligned}
\iint D_{z_{\mathrm{o}}} \Omega d S & =2 \pi a \sum_{\mathrm{s}=0}^{\infty}(-1)^{\mathrm{s}} \frac{\left(\frac{a D_{\mathrm{z}_{\mathrm{o}}}}{2}\right)^{2 \mathrm{~s}+1}}{s !(s+1) !} \Omega^{\left(x_{0}, y_{0}, z_{\mathrm{o}}\right)} \\
& =2 \pi a J_{1}\left(a D_{\mathrm{z}_{\mathrm{o}}}\right) \Omega\left(x_{\mathrm{o}}, y_{\mathrm{o}}, z_{\mathrm{o}}\right)
\end{aligned}
$$


If $\Omega$ represents the magnetic, scalar, potential whose negative gradient is a magnetic field, then the magnetic flux through the circle may be written in the symbolic form

$$
M \equiv-\int \mathcal{S} D_{\mathrm{n}} \Omega d S=-2 \pi a_{2} J_{1}\left(a_{2} D_{\mathrm{n}_{2}}\right) \Omega\left(x_{2}, y_{2}, z_{2}\right)
$$

where $a_{2}$ is the radius of the circle, $P_{2}\left(x_{2}, y_{2}, z_{2}\right)$ its center and $D_{\mathbf{n}_{2}} \Omega$ represents the value at $P_{2}$ of the space derivative of $\Omega$ in the direction of the positive normal to the plane of the circle, which is such that the positive direction of the current encircles it right-handedly. It is evident that $M$ is harmonic in the variables $x_{2}, y_{2} z_{2}$, if the orientation of the circle is held constant; that is, $\left(D_{\mathrm{x} 2}^{2}+D_{\mathrm{y}_{2}}^{2}+D_{\mathrm{x}_{2}}^{2}\right) M=0$.

\section{SYMBOLIC EXPRESSION FOR THE MAGNETIC POTEN- TIAL OF A CIRCULAR CURRENT}

If there is a unit current in another circle of radius $a_{1}$, whose plane is in the $y z$ plane and whose positive normal is the positive $x$ axis so that its center is at the origin of coordinates, then the (scalar) magnetic potential $\Omega(x, y, z)$ due to it at any point $P(x, y, z)$ may be found from the fact that it is harmonic and is a function of the two cylindrical coordinates only, namely, $x$ and $\rho=\sqrt{y^{2}+z^{2}}$, and reduces when $x=+0$ to 0 if $\rho>a_{1}$ and to $2 \pi$ if $\rho<a_{1}$. (This is evident since $\Omega$ is the solid angle subtended at $P$ by the positive area of the circle.) From the known discontinuous function defined by the integral

$$
\begin{aligned}
2 \pi a_{1} \int_{0}^{\infty} J_{1}\left(a_{1} s\right) J_{0}(\rho s) d s & =0 \text { if } \rho>a_{1} \\
& =2 \pi \text { if } \rho<a_{1}
\end{aligned}
$$

and from the fact that a function of $x$ and $\rho$ only, which is a particular solution of Laplace's equation, is $\boldsymbol{e}^{ \pm \mathrm{Bx}} J_{0}(\rho s)$, it is readily seen that

where

$$
\begin{aligned}
\Omega(x, \rho) & =2 \pi a_{1} \int_{0}^{\infty} e^{-8 x} J_{1}\left(a_{1} s\right) J_{0}(\rho s) d s \text { if } x>0 \\
& =-2 \pi a_{1} \int_{0}^{\infty} e^{\mathrm{gx}} J_{1}\left(a_{1} s\right) J_{0}(\rho s) d s \text { if } x<0
\end{aligned}
$$

The expression for $\Omega$ may be given another form by using the formula

$$
\int_{0}^{\infty} e^{-\mathbf{s}|\mathbf{x}|} J_{0}\left(s \sqrt{y^{2}+z^{2}}\right) d s=\frac{1}{\sqrt{x^{2}+y^{2}+z^{2}}}
$$

and by noting that, since

$$
\begin{gathered}
\left(-a_{1} D_{\mathbf{x}}\right)^{\mathrm{n}} e^{-\mathrm{sx}}=\left(a_{1} s\right)^{\mathrm{n}} e^{-\mathrm{sx}} \\
-J_{1}\left(a_{1} D_{\mathrm{x}}\right) e^{-\mathrm{sx}}=J_{1}\left(-a_{1} D_{\mathbf{x}}\right) e^{-\mathrm{sx}}=e^{-\mathrm{sx}} J_{1}\left(a_{1} s\right)
\end{gathered}
$$


and consequently

$$
\begin{gathered}
\int_{0}^{\infty} e^{-\mathrm{sx}} J_{1}\left(a_{1} s\right) J_{\mathrm{o}}(\rho s) d s=-J_{1}\left(a_{1} D_{\mathrm{x}}\right) \int_{0}^{\infty} e^{-\mathrm{sx}} J_{0}(\rho s) d s \\
=-J_{1}\left(a_{1} D_{\mathrm{x}}\right) \frac{1}{\sqrt{x^{2}+y^{2}+z^{2}}}
\end{gathered}
$$

Consequently, the two expressions (12) for $\Omega$ may be put in either of the following forms, each of which holds for both positive and negative values of $x$, namely

$$
\begin{aligned}
\Omega(x, \rho) & =-2 \pi a_{1} J_{1}\left(a_{1} D_{\mathbf{x}}\right) \frac{1}{\sqrt{x^{2}+y^{2}+z^{2}}} \\
& =-2 \pi a_{1} J_{1}\left(a_{1} D_{\mathbf{x}}\right) \int_{0}^{\infty} e^{-s|\mathbf{x}|} J_{\mathrm{o}}(\rho s) d s
\end{aligned}
$$

Remembering the special relation of this circle to the $x$ axis, it is easy to write out from a consideration of equation (15) the expression for the value of the magnetic potential $\Omega$ at a point $P_{2}\left(x_{2}, y_{2}, z_{2}\right)$ due to unit current in a circle of radius $a_{1}$ with center at $P_{1}\left(x_{1}, y_{1}, z_{1}\right)$, whose plane has the positive normal $n_{1}$. It is

$$
\Omega\left(x_{2}, y_{2}, z_{2}\right)=2 \pi a_{1} J_{1}\left(a_{1} D_{\mathbf{n}_{1}}\right) \frac{1}{\sqrt{\left(x_{2}-x_{1}\right)^{2}+\left(y_{2}-y_{1}\right)^{2}+\left(z_{2}-z_{1}\right)^{2}}}
$$

where $a_{1} D_{\mathrm{n}_{1}}$ represents the directional derivative in the direction $n_{1}$, with respect to the variables $x_{1}, y_{1}, z_{1}$; that is, if $l_{1}, m_{1}, n_{1}$ are the direction cosines of the positive normal to this circle

$$
a_{1} D_{\mathrm{n}_{1}}=a_{1}\left(l_{1} D_{\mathrm{x}_{1}}+m_{1} D_{\mathrm{y}_{1}}+n_{1} D_{\mathrm{z}_{1}}\right)
$$

\section{COMBINATION OF II AND III FOR THE MUTUAL IN- DUCTANCE OF TWO CIRCLES}

Equation (16) is purley formal, like equation (10), to which it bears a certain resemblance. Combining the two, leads to the following symbolic formula for the mutual inductance of any two circles of radii $a_{1}$ and $a_{2}$ with positive normals $n_{1}$ and $n_{2}$.

$$
M=-4 \pi^{2} a_{1} a_{2} J_{1}\left(a_{1} D_{\mathrm{n} 1}\right) J_{1}\left(a_{2} D_{\mathrm{n} 2}\right) \frac{1}{\sqrt{\left(x_{2}-x_{1}\right)^{2}+\left(y_{2}-y_{1}\right)^{2}+\left(z_{2}-z_{1}\right)^{2}}}
$$

where $P_{1}\left(x_{1}, y_{1}, z_{1}\right)$ and $P_{2}\left(x_{2}, y_{2}, z_{2}\right)$ are the centers of the circles, respectively, and $n_{1}, n_{2}$ are unit vectors normal to their positive sides.

\section{CASE OF PARALLEL CIRCLES}

In the special case where the circles are parallel, the first one may be taken in the $y z$ plane with its center at the origin, the center of the 
second in the $x y$ plane. Denoting the coordinates of the second circle by $x y$ (or polar coordinate $r, \theta$ ), the formula (17) gives

$$
\begin{aligned}
M & =4 \pi^{2} a_{1} a_{2} J_{1}\left(a_{1} D_{\mathbf{x}}\right) J_{1}\left(a_{2} D_{\mathbf{x}}\right) \frac{1}{r} \\
& =4 \pi^{2} a_{1} a_{2} \int_{0}^{\infty} e^{-s|\mathbf{x}|} J_{0}(y s) J_{1}\left(a_{1} s\right) J_{1}\left(a_{2} s\right) d s \\
& =-4 \pi^{2} a_{2}{ }_{2} \sum_{n=1}^{\infty}(-1)^{\mathrm{n}} \frac{F\left(-n,-n+1,2, \frac{a^{2}}{a^{2}}\right)}{(n-1) ! n !}\left(\frac{a_{1}}{2}\right)^{2 \mathrm{n}} D^{2 \mathrm{n}} \times \frac{1}{r}
\end{aligned}
$$

where $F$ denotes the hypergeometric function. But

so that

$$
D^{2 \mathrm{n}} \frac{1}{r}=\frac{(2 n) !}{r^{2 \mathrm{n}+1}} P_{2 \mathrm{n}}(\cos \theta)=\frac{2^{2 \mathrm{n}}}{\sqrt{\pi}} \frac{n ! \Gamma\left(n+\frac{1}{2}\right)}{r^{2 \mathrm{n}+1}} P_{2 \mathrm{n}}(\cos \theta)
$$

$$
\begin{gathered}
M=-\frac{4 \pi^{2} a_{2}^{2}}{\sqrt{\pi} a_{1}} \sum_{\mathrm{n}=1}^{\infty}(-1)^{\mathrm{n}}\left(\frac{a_{1}}{r}\right)^{2 \mathrm{n}+1} P_{2 \mathrm{n}}(\cos \theta) . \\
\cdot \frac{\Gamma\left(n+\frac{1}{2}\right)}{(n-1) !} F\left(-n,-n+1,2, \frac{a_{2}^{2}}{a_{1}^{2}}\right)
\end{gathered}
$$

which converges for large values of $r$. This is identical with a formula obtained by Butterworth. ${ }^{2}$

Formula (18) is valid when $r>a_{1}+a_{2}$.

In the other extreme, where $r<a_{1}-a_{2}$, one finds

$$
\begin{gathered}
M=\frac{4 \pi^{3 / 2} a_{2}^{2}}{a_{1}} \sum_{n=0}^{\infty}(-1)^{\mathrm{n}} \frac{\Gamma\left(n+\frac{3}{2}\right)}{n !} \\
F\left(n+\frac{1}{2}, n+\frac{3}{2}, 2, \frac{a_{2}^{2}}{a_{1}^{2}}\right)\left(\frac{r}{a_{1}}\right)^{2 \mathrm{n}} P_{2 \mathrm{n}}(\cos \theta)
\end{gathered}
$$

This may be derived from (17) by writing the latter in the form

$$
\begin{aligned}
M & =-4 \pi^{2} a_{1} a_{2} J_{\mathrm{o}}\left(y D_{\mathrm{x}}\right) J_{1}\left(a_{2} D_{\mathrm{x}}\right) \int_{0}^{\infty} e^{-\mathrm{sx}} J_{1}\left(a_{1} s\right) d s \\
& =4 \pi^{2} a_{2} J_{\mathrm{o}}\left(y D_{\mathrm{x}}\right) J_{1}\left(a_{2} D_{\mathrm{x}}\right) \frac{x}{\sqrt{a^{2}+x^{2}}}
\end{aligned}
$$

by using the binomial expansion

$$
\frac{x}{\sqrt{a^{2}+x^{2}}}=\frac{1}{\sqrt{\pi}} \sum_{k=0}^{\infty}(-1)^{\mathbf{k}} \frac{\Gamma\left(k t \frac{1}{2}\right)}{k !}\left(\frac{x}{a_{1}}\right)^{2 \mathbf{k}+1}
$$

${ }^{2}$ See footnote 1, p. 531 . 
together with the operational expansion

$$
\begin{gathered}
J_{\mathrm{o}}\left(y D_{\mathrm{x}}\right) J_{1}\left(a_{2} D_{\mathrm{x}}\right)=-\frac{a_{2}}{2} \sum_{n=0}^{\infty}(-1)^{\mathrm{n}} \frac{\left(\frac{a_{2}}{2}\right)^{2 \mathrm{n}}}{n !(n+1) !} \\
F\left(-n,-n-1,1, \frac{y^{2}}{a_{2}^{2}}\right) D_{\mathbf{x}}^{2 \mathrm{n}+1}
\end{gathered}
$$

\section{SPECIAL CASE WHERE THE AXES OF THE TWO CIRCLES INTERSECT}

A considerably more general case (but not the most general) is that in which the axes of the two circles intersect. Taking the plane of these two axes as the $x y$ plane, with the origin at the point of intersection of the two axes, the center of the first circle being on the $x$ axis with abscissa $x_{1}\left(x_{1}\right.$ may be taken as never negative without loss of generality), and that of the second circle having the plane polar coordinates $r, \theta$, then (17) becomes (see fig. 1)

$$
\begin{aligned}
M & =-2 \pi a_{2} J_{1}\left(a_{2} D_{\mathrm{r}}\right) \Omega(r, \theta) \\
& =-2 \pi a_{2} J_{1}\left(a_{2} D_{\mathrm{r}}\right) 2 \pi a_{1} J_{1}\left(a_{1} D_{\mathrm{x}_{1}}\right) \frac{1}{\sqrt{x^{2}{ }_{1}-2 x_{1} r \cos \theta+r^{2}}}
\end{aligned}
$$

There is no loss of generality in taking for circle No. 1 that one of the two whose center is most distant from the origin. Hence, we may use the expansion

$$
\frac{1}{\sqrt{x_{1}^{2}-2 x_{1} r \cos \theta+r^{2}}}=\sum_{\mathrm{s}=0}^{\infty} \frac{r^{\mathrm{s}}}{x_{1}{ }^{8+1}} P_{\mathrm{s}}(\cos \theta) \text { since } x_{1}>r
$$

where $P_{\mathrm{s}}$ is Legendre's coefficient. Applying to this expansion the symbolic formula (16) one finds

$$
\begin{aligned}
& \Omega(r, \theta)=2 \pi a_{1} J_{1}\left(a_{1} D_{\mathrm{x}_{1}}\right) \frac{1}{\sqrt{x^{2}{ }_{1}-2 x_{1} r \cos \theta+r^{2}}} \\
& =2 \pi a_{1} \sum_{\mathbf{k}=0}^{\infty} \frac{(-1)^{\mathbf{k}}\left(\frac{a_{1}}{2}\right)^{2 \mathrm{k}+1}}{k !(k+1) !} D_{\mathbf{x} 1}{ }^{2 \mathrm{k}+1} \frac{1}{\sqrt{x_{1}^{2}-2 x_{1} r \cos \theta+r^{2}}} \\
& =-\frac{\pi a_{1}^{2}}{x_{1}{ }_{1}} \sum_{\mathrm{k}=0}^{\infty} \sum_{\mathrm{s}=0}^{\infty} \frac{(-1)^{\mathrm{k}} \Gamma(2 k+s+2)}{\Gamma(k+1) \Gamma(k+2) \Gamma(s+1)}\left(\frac{a_{1}}{2 x_{1}}\right)^{2 \mathrm{k}}\left(\frac{r}{x_{1}}\right)^{\mathrm{s}} P_{\mathrm{s}}(\cos \theta) \\
& =-\left[\frac{\pi a^{2}}{x^{2}} \sum_{s=0}^{\infty}\left(\frac{r}{x_{1}}\right)^{\mathrm{s}} P_{\mathrm{s}}(\cos \theta) \frac{2^{2 \mathrm{~s}+1}}{\sqrt{\pi} \Gamma(s+1)}\right] \text {. } \\
& \cdot\left[\sum_{\mathrm{k}=0}^{\infty} \frac{\Gamma\left(k+\frac{s+2}{2}\right) \Gamma\left(k+\frac{s+3}{2}\right)}{\Gamma(k+1) \Gamma(k+2)}\left(-\frac{a_{1}^{2}}{x^{2}}\right)^{\mathrm{k}}\right] \\
& =-\frac{\pi a_{1}^{2}}{x_{1}^{2}} \sum_{\mathrm{s}=0}^{\infty}\left(\frac{r}{x_{1}}\right)^{\mathrm{s}}(s+1) P_{\mathrm{s}}(\cos \theta) F\left(\frac{s+2}{2}, \frac{s+3}{2}, 2,-\frac{a_{1}^{2}}{x_{1}^{2}}\right)
\end{aligned}
$$


where

$$
=-\pi \sin ^{2} a_{1} \sum_{s=0}^{\infty}\left(\frac{r}{r_{1}}\right)^{s}(s+1) P_{s}(\cos \theta) F\left(\frac{2+s}{2}, \frac{1-s}{2}, 2, \sin ^{2} \alpha_{1}\right)
$$

$$
\tan \alpha_{1}=\frac{a_{1}}{x_{1}} \text { and } r_{1}^{2}=x_{1}^{2}+a_{1}^{2}
$$

Now by Gauss's transformation one finds that

$$
\begin{aligned}
F\left(\frac{2+s}{2}, \frac{1-s}{2}, 2, \sin ^{2} \alpha_{1}\right) & =\frac{2\left(1-\cos \alpha_{1}\right)}{\sin ^{2} \alpha_{1}} \text { if } s=0 \\
& =\frac{2 P^{\prime}{ }_{8}\left(\cos \alpha_{1}\right)}{s(s+1)} \text { if } s=1,2,3, \ldots \ldots
\end{aligned}
$$

where $P^{\prime}{ }_{\mathrm{s}}$ denotes the derivative of $P_{\mathrm{s}}$ with respect to its argument. Using (21) in the preceding expression for $\Omega$ gives

$$
\Omega(r, \theta)=-2 \pi\left(1-\cos \alpha_{1}\right)-2 \pi \sin ^{2} \alpha_{1} \sum_{s=1}^{\infty}\left(\frac{r}{r_{1}}\right)^{\mathrm{s}} \frac{P_{\mathrm{B}}(\cos \theta) P_{\mathrm{B}}^{\prime}\left(\cos \alpha_{1}\right) \quad(22)}{s}
$$

a formula which holds when the origin is any point on the axis, to the left of the circle if $r<r_{1}$. Substituting this value of $\Omega$ in the symbolic formula (19) gives

$$
\begin{aligned}
M & =4 \pi^{2} a_{2} \sin ^{2} \alpha_{1} \sum_{\mathrm{k}=0}^{\infty} \frac{(-1)^{\mathrm{k}}\left(\frac{a_{2}}{2}\right)^{2 \mathrm{k}+1} D_{\mathrm{r}}{ }^{2 \mathrm{k}+1}}{k !(k+1) !} \sum_{\mathrm{s}=1}^{\infty} \frac{r^{\mathrm{s}} P_{\mathrm{B}}(\cos \theta) P_{\mathrm{s}}\left(\cos \alpha_{1}\right)}{s r_{1}^{\mathrm{B}}} \\
& =4 \pi^{2} a_{2} \sin ^{2} \alpha_{1} \sum_{\mathrm{k}=0}^{\infty} \sum_{\mathrm{s}=1}^{\infty} \frac{(-1)^{\mathrm{k}}\left(\frac{a_{2}}{2 r}\right)^{2 \mathrm{k}+1}(s-1) !}{k !(k+1) ! \Gamma(s-2 k)}\left(\frac{r}{r_{1}}\right)^{\mathrm{s}} P_{\mathrm{s}}(\cos \theta) P^{\prime}\left(\cos \alpha_{1}\right) \\
& =\frac{2 \pi^{2} \alpha_{2}{ }^{2} \sin ^{2} \alpha_{1}}{r} \sum_{\mathrm{s}=1}^{\infty}\left(\frac{r}{r_{1}}\right)^{\mathrm{s}} P_{\mathrm{s}}(\cos \theta) P_{\mathrm{s}}^{\prime}\left(\cos \alpha_{1}\right) \Gamma(s) \sum_{\mathrm{k}=0}^{\infty} \frac{(-1)^{\mathrm{k}}\left(\frac{a_{2}}{2 r}\right)^{2 \mathrm{k}}}{\Gamma(k+1) \Gamma(k+2) \Gamma(s-2 k)}
\end{aligned}
$$

or

where

$$
M=\frac{4 \pi^{2} a_{1}^{2} a_{2}^{2}}{r_{1}^{2} r_{2}} \sum_{s=1}^{\infty}\left(\frac{r_{2}}{r_{1}}\right)^{s} \frac{P_{8}(\cos \theta) P_{s}^{\prime}\left(\cos \alpha_{1}\right) P_{s}^{\prime}\left(\cos \alpha_{2}\right)}{s(s+1)}
$$

$$
\begin{aligned}
& r_{1}^{2}=x_{1}^{2}+a_{1}^{2}, \tan \alpha^{1}=\frac{a_{1}}{x_{1}} \\
& r_{2}{ }_{2}=r^{2}+a_{2}^{2}, \tan \alpha_{2}=\frac{a_{2}}{r}
\end{aligned}
$$

$\alpha_{1}$ and $\alpha_{2}$ are positive acute angles. 
The formula (23) gives the mutual inductance between any two circles of radii $a_{1}$ and $a_{2}$ whose axes intersect. The circle No. 1 is chosen as that one of the two whose center is most distant from the origin. The origin is the point of intersection of the two axes and $\theta$ the angle between these axes and may be anywhere in the range from zero to $\pi$. The origin is taken as lying to the left of the circle No. 1 whose center is on the $x$ axis at $x=x_{1}$. The distance of the center of the second circle from the origin is $r$. (See fig. 1 where $r=\overline{O C_{2}}$.)

(a) The Axes Intersect at Center of One Circle.-A special case of (23) is that in which the center of the second circle $C_{2}$ coincides

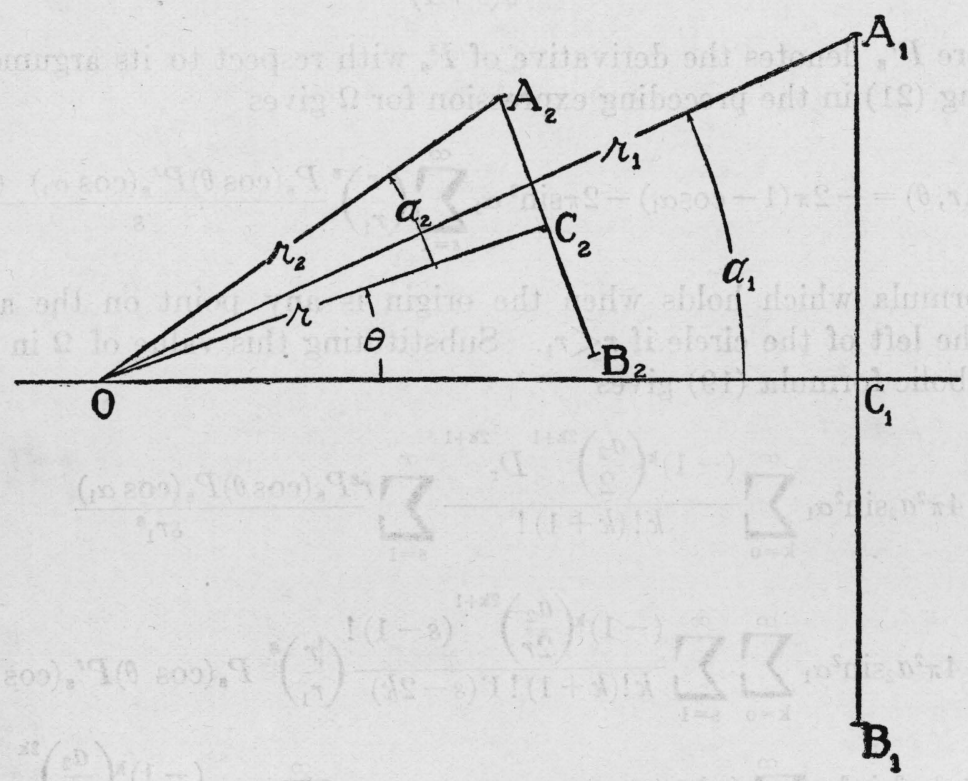

FIG. 1.-A principal section of two circles whose axes intersect

with $O$ (fig. 1 ) so that $r_{2}$ becomes $a_{2}$ and $\alpha_{2}$ becomes $\frac{\pi}{2}$. In this case $P_{s}^{\prime}\left(\cos a_{2}\right)$ vanishes when $s$ is an even integer, and if $s$ is an odd integer, say $2 n+1$, reduces to $\frac{2}{\sqrt{\pi}}(-1)^{\mathrm{n}} \frac{\Gamma\left(n+\frac{3}{2}\right)}{n !}$

so that (23) reduces to

$\left.M=2 \pi_{1} \sin \alpha_{1} \sqrt{\pi} \sum_{n=0}^{\infty}(-1)^{\mathrm{n}}\left(\frac{a_{2}}{r_{1}}\right)^{2 \mathrm{n}+2} \frac{\Gamma\left(n+\frac{1}{2}\right)}{(n+1) !} P_{2 \mathrm{n}+1}(\cos \theta) P_{2 \mathrm{n}+1}^{\prime} \cos \alpha_{1}\right)$ 
(b) Circles Coaxial.- Specializing this still further by making the circles coaxial (so that $\mu=\cos \theta=1$ ) gives

$$
M=2 \pi a_{1} \sin \alpha_{1} \sqrt{\pi} \sum_{n=0}^{\infty}(-1)^{\mathrm{n}}\left(\frac{a_{2}}{r_{1}}\right)^{2 \mathrm{n}+2} \frac{\Gamma\left(n+\frac{1}{2}\right)}{(n+1) !} \mathrm{P}_{2 \mathrm{n}+1}^{\prime}\left(\cos \alpha_{1}\right)
$$

when

$$
\frac{a_{2}}{r_{1}}<1
$$

This is easily shown to be reducible to Maxwell's formula involving elliptic integrals.

(c) Circles Concentric, but not Necessarily Coaxial.Specializing (23a) in another direction, by letting the circles become concentric $\left(a_{2}\right.$ being less than $\left.a_{1}\right)$, gives

$$
M=\frac{4 \pi a_{2}^{2}}{a_{1}} \sum_{n=0}^{\infty}\left(\frac{a_{2}}{a_{1}}\right)^{2 \mathrm{n}} \Gamma \frac{\left(n+\frac{1}{2}\right) \Gamma\left(n+\frac{3}{2}\right)}{n !(n+1) !} P_{2 \mathrm{n}+1}(\cos \theta)
$$

Thus, by considerations of continuity, we have arrived by transformations at a formula which is valid, where the original assumption with which we started is not satisfied, namely, that the field of $a_{2}$ is expressible by Taylor's theorem over a sphere of radius $a_{1}$.

\section{CONVERGENCE OF THE EXPANSION}

Returning to a consideration of the general formula (23), it is readily seen that this series converges when $r_{2}<r_{1}$, so that by interchanging $r_{2}$ and $r_{1}$ a formula is obtained for the case when $r_{2}>r_{1}$. The proof of the absolute convergence of this series may be based upon the fact that $P_{\mathrm{s}}$ is never greater than unity when its argument is the cosine of a real angle and upon the fact that

$$
P_{\mathrm{B}}^{\prime}(\cos \alpha)=\frac{s(s+1)}{s+\frac{1}{2}} \frac{P_{\mathrm{B}-}(\cos \alpha)-P_{\mathrm{B}+1}(\cos \alpha)}{2 \sin ^{2} \alpha}
$$

which shows that

$$
\begin{aligned}
& \left|\frac{P_{\mathrm{B}}(\cos \theta) P_{\mathrm{B}}^{\prime}\left(\cos \alpha_{1}\right) P_{\mathrm{B}}^{\prime}\left(\cos \alpha_{2}\right)}{s(s+1)}\right|= \\
= & \left.\mid \frac{P_{\mathrm{B}}(\cos \theta)}{1+\frac{1}{4 s(s+1)}}\right]\left[\frac{P_{\mathrm{B}-1}\left(\cos \alpha_{1}\right)-P_{\mathrm{s}+1}\left(\cos \alpha_{1}\right)}{2 \sin ^{2} \alpha_{1}}\right]\left[\frac{P_{\mathrm{B}-1}\left(\cos \alpha_{2}\right)-P_{\mathrm{B}+1}\left(\cos \alpha_{2}\right)}{2 \sin ^{2} \alpha_{2}}\right] \\
\leqq & \frac{1}{\sin ^{2} \alpha_{1} \sin ^{2} \alpha_{2}}=\frac{r_{1}^{2} \dot{r}_{2}{ }_{2}}{a^{2}{ }_{1} a^{2}{ }_{2}}
\end{aligned}
$$


Hence, if $r_{2}<r_{1}$

$$
M \leqq 4 \pi^{2} r_{2} \sum_{s=1}^{\infty}\left(\frac{r_{2}}{r_{1}}\right)^{\mathrm{s}}=\frac{4 \pi^{2} r_{1}{ }^{2}}{r_{1}-r_{2}}
$$

No discussion of the convergence range of the symbolic formula is possible without first choosing the type of coordinates suitable for performing the series of differentiations. The convergence range usually makes itself evident in the process of performing the expansions.

Washington, May 19, 1928. 